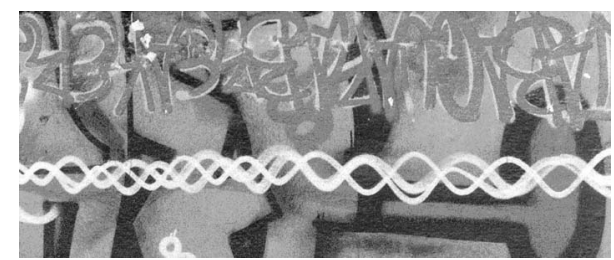

\title{
Comunicação e informação em Saúde no pré-natal*
}

Escolástica Rejane Ferreira Moura ${ }^{1}$ Maria Socorro Pereira Rodrigues ${ }^{2}$

MOURA, E. R. F.; RODRIGUES, M. S. P. Pre natal healthcare communication and information, Interface Comunic, Saúde, Educ, v.7, n.13, p.109-18, 2003.

This investigation intended to examine the business of healthcare communication and information, during prenatal care carried out by nurses working in the Family Health Program (HFP) in Brazil. The data were collected in eight towns in Ceará state from May to June of 2001, using interviews, a questionnaire and participant observation. Thirty nurses and thirty pregnant women constituted the sample. It was observed that healthcare communication and information activities were implemented by the group in a more participative and innovative way, in the universe surveyed, therefore, it was performed less frequently. The absence of planning the group activities was observed, thus systematically and continuously jeopardizing the execution. It was seen that individual nurses have undertaken healthcare communication and information activities as part of routine care of pregnant women, their performance recognized by the government. These activities should be implemented in such a way as to replace medical consultations, with the purpose of helping the introduction of a healthcare promotion model.

KEY WORDS: Prenatal care; primary health care; health promotion; Health Education; communication.

Trata-se de pesquisa que teve por objetivo investigar as atividades de comunicação/informação em saúde, implementadas no decorrer da assistência pré-natal, por enfermeiros que atuam no Programa Saúde da Família, Brasil (PSF). Os dados foram colhidos em oito municípios do Estado do Ceará, de maio a junho de 2001, utilizando as técnicas de observação participante, entrevista e questionário. A amostra foi constituída por trinta enfermeiros e trinta gestantes. Constatou-se que as atividades de comunicação/informação em saúde começam a ser implementadas de forma mais inovadora e participativa, no universo pesquisado, porém com baixa freqüência. Identificou-se ausência de planejamento das atividades grupais, prejudicando sua execução de maneira sistemática e contínua. Já no âmbito individual, os enfermeiros estão desenvolvendo ações de comunicação/ informação como parte da rotina dos cuidados de enfermagem às gestantes, inclusive tendo seus desempenhos reconhecidos no âmbito estadual. Recomenda-se que essas atividades sejam implementadas de maneira a sobrepor as consultas, a fim de contribuir para a implantação de um modelo de promoção da saúde.

PALAVRAS-CHAVE: Cuidado pré-natal; cuidados primários de saúde; promoção da saúde; Educação em Saúde; comunicação.

\footnotetext{
*Artigo produzido a partir de Dissertação de Mestrado (Moura, 2001).

${ }^{1}$ Enfermeira, Assessora Técnica da Secretaria da Saúde do Estado do Ceará, Brasil. <escolpaz@yahoo.com.br>

${ }^{2}$ Enfermeira, Professora Adjunta da Universidade Federal do Ceará, Brasil. <socorro@ufc.br>
} 


\section{Introdução}

A década de 1980 foi marcada como um período de transição democrática no qual um crescente sentimento de democratização tomou conta do país, fazendo despontar a organização de vários movimentos sociais, destacando-se o movimento feminista. As mulheres passaram a reivindicar direitos relacionados à saúde reprodutiva, como o planejamento familiar, a sexualidade, a democratização da informação em saúde, além de outras medidas entendidas na esfera da Saúde Pública e não mais, na esfera biomédica. Nesse período, a saúde da mulher passou a ser discutida com mais intensidade nas universidades, nos sistemas formais de saúde e nas organizações sociais (Tyrrel \& Carvalho, 1995). As mesmas autoras afirmam que, em decorrência desse processo de mobilização social e de formação de alianças com as instituições formais de saúde, o Ministério da Saúde (MS), em conjunto com líderes feministas e profissionais da saúde, iniciaram a elaboração de normas e propostas capazes de garantir à mulher o atendimento a seus direitos reprodutivos, resultando na estruturação das bases fundamentais do Programa de Assistência Integral à Saúde da Mulher (PAISM). A implantação do PAISM veio estimular o desenvolvimento de várias atividades de cunho informativo/educativo nas unidades de saúde, com vistas à promoção do autoconhecimento e da autoestima das mulheres, em que se incluíram oficinas e outros tipos de abordagens grupais com enfoque participativo.

Sobre a assistência pré-natal, o MS enfatiza que a gestação caracteriza-se por ser um período de mudanças físicas e emocionais, determinando que o principal objetivo do acompanhamento pré-natal seja o acolhimento à mulher, o oferecimento de respostas e de apoio aos sentimentos de medo, dúvidas, angústias, fantasias ou, simplesmente, à curiosidade de saber sobre o que acontece com o seu corpo (Brasil, 2000). Segundo Navajas Filho (1997), o mais importante para a equipe e, em particular, para o enfermeiro que presta cuidado às gestantes no pré-natal, é conhecer o que está acontecendo com elas e saber que, por trás de toda pergunta, aparentemente ingênua, feita por uma gestante, poderão existir importantes demandas emocionais latentes. Infere-se, portanto, que o estabelecimento de uma escuta ativa, aliada a uma prática de comunicação/informação adequada junto às gestantes, parece contribuir sobremaneira para que essas mulheres ganhem autonomia, passando a participar da promoção de sua saúde e da saúde do concepto.

As atividades de comunicação/informação em saúde devem ser priorizadas no transcurso da assistência pré-natal, uma vez que o intercâmbio de informações e experiências pode ser a melhor forma de promover a compreensão do processo da gestação. Neste sentido, o MS ressalta que o foco principal do processo de orientação/informação deve ser as gestantes, incluindo, porém, seus companheiros e familiares (Brasil, 2000). Sendo assim, o contexto em que se apresenta o Programa Saúde da Família (PSF) parece favorecer uma práxis efetiva de comunicação/informação em saúde, visto ter como prioridade a promoção da saúde e prevenção das doenças, cujo pilar de sustentação é exatamente a educação em saúde. Ademais, os profissionais do PSF compreendem que seu universo de atuação é a pessoa 
inserida no seio familiar e integrada a uma comunidade. Sobre esse assunto Varela (2000) considera fundamental o envolvimento dos profissionais de saúde com outros atores da comunidade (familiares, lideranças etc.). A autora ressalta que esses atores levam cuidados de saúde à população dentro do sistema comunitário, exercendo forte influência, aspecto que precisa ser levado em conta pelos profissionais. Portanto, a aproximação entre enfermeiros e esses atores sociais parece ser um elemento facilitador à adequada compreensão da realidade e identificação dos traços culturais da comunidade.

Diante do exposto, decidiu-se pela realização do presente trabalho, tendo por objetivo realizar uma análise crítica das atividades de comunicação/ informação em saúde, implementadas pelos enfermeiros, por ocasião da assistência pré-natal, oferecida no PSF de oito municípios do Ceará.

Materiais e métodos

Trata-se de pesquisa descritiva, com abordagem predominantemente qualitativa. Os dados foram coletados em oito municípios que compõem a micro região de saúde de Baturité, Ceará, no período de abril a julho de 2001.

A amostra foi constituída por trinta enfermeiros e trinta gestantes. $\mathrm{O}$ número de enfermeiros correspondeu a $93,8 \%$ do total de enfermeiros da micro região que atuavam no PSF, uma vez que dois se eximiram da pesquisa. $\mathrm{O}$ número de gestantes foi determinado pela saturação dos comentários abordados nas entrevistas. Para a seleção dos sujeitos, foram estabelecidos os seguintes critérios: as enfermeiras deveriam estar atuando na assistência pré-natal, enquanto integrantes de equipe de PSF, $e$ manifestar aceitação voluntária à participação no estudo; as gestantes deveriam ter realizado, pelo menos, três consultas de enfermagem no prénatal atual, e demonstrar aceitação voluntária à participação na pesquisa.

A coleta dos dados procedida junto às gestantes foi realizada por meio de entrevista, com base em roteiro pré-estabelecido. Foram realizadas, em sua maioria, nas Unidades Básicas de Saúde da Família (UBASF) e as demais, no domicílio das próprias gestantes. Esta tarefa contou com a colaboração de enfermeiras, agentes de saúde e auxiliares de enfermagem no sentido de guiar e acompanhar a pesquisadora aos referidos domicílios. Segundo Cruz Neto (1995, p.57), a entrevista "faz parte da relação mais formal do trabalho de campo, em que, intencionalmente, o pesquisador recolhe informações, através da fala dos atores sociais”. Para este autor a entrevista é o procedimento mais usual no trabalho de campo, para obtenção de dados objetivos e subjetivos sobre determinada temática. Optou-se pela modalidade de entrevista semi-estruturada, por esta permitir, também, que o informante aborde, livremente, o tema proposto, sem respostas ou condições prefixadas pelo pesquisador.

O levantamento de dados junto às enfermeiras foi realizado mediante solicitação de respostas diretas ao questionário, em caráter de autoaplicação. Neste sentido, é importante informar que, em princípio, foi testado tanto o roteiro de entrevista para as gestantes como um roteiro de entrevista para as enfermeiras, utilizando-se um gravador como recurso 
para documentar as falas. Foram entrevistadas, na ocasião, cinco enfermeiras $e$ oito gestantes ${ }^{3}$. Levando-se em conta o fato de que as enfermeiras se encontravam no ambiente de trabalho, com uma alta demanda de atendimento e considerando que as UBASF não ofereciam um ambiente adequado para gravação, decidiu-se pelo uso do questionário auto-aplicável pelas próprias enfermeiras e o registro escrito pela pesquisadora, no caso das gestantes.

Utilizou-se a observação participante, por ocasião da interação com coordenadores de PSF, funcionários das UBASF, enfermeiras, famílias e gestantes, buscando aprofundamento para compreensão do objeto estudado. Para Triviños (1993), a observação ajuda a conhecer os atos, a dinâmica espontânea dos indivíduos, sua prática, e seu cotidiano, possibilitando o entendimento do fenômeno que está sendo investigado. Os dados advindos da observação foram registrados em um diário de campo, com o objetivo de documentar esta etapa da pesquisa, com relação a tudo o que se passava no contexto social. Durante a fase de coleta de dados teve-se a oportunidade de participar de dois seminários micro regionais (Seminário Micro regional da Atenção Primária e Seminário Micro regional de Saúde da Mulher), e da Semana de Enfermagem, quando houve possibilidade de constatar a dimensão da educação continuada das enfermeiras, de aspectos de saúde trazidos para debate, favorecendo a interação das pesquisadoras com as enfermeiras e enriquecendo o diário de campo.

\section{Resultados e discussão}

Os enfermeiros afirmaram que as atividades de comunicação/informação em saúde dirigidas às gestantes são realizadas no transcorrer das consultas de enfermagem (nível individual) e, também, por meio de atividades grupais. Outra forma citada foi por ocasião das reuniões mensais, às quais a gestante comparece com objetivo específico e, visto serem mais prolongadas, favorecem melhor captação das mensagens divulgadas.

As principais temáticas abordadas pelos enfermeiros, por ocasião da assistência pré-natal, quer seja no nível individual (com maior freqüência) e/ou grupal, $e$ das quais emergem as informação em saúde, conforme afirmam as gestantes, destacaram-se: preparação para o parto, cuidado com as mamas $e$ preparo para o aleitamento materno, vestuário adequado, combate ao tabagismo, uso de medicamentos, alimentação e cuidados com a criança (higiene e vacinação), exames laboratoriais, atividade física regular, contato $e$ afeto com o bebê, ainda no útero, entre outros.

Diante desses dados, constatou-se que os enfermeiros do universo estudado parecem ser conhecedores da importância da prática de informação em saúde, principalmente no nível individual, e que as temáticas abordadas possam ajudar a gestante a se conduzir de forma mais adequada no que diz respeito às circunstâncias da gestação, do parto e puerpério. A avaliação de impacto da situação da Saúde Reprodutiva no Ceará (Ceará, 1998) divulgou que os enfermeiros vêm estabelecendo satisfatória interação com as gestantes $e$ proporcionando a essas mulheres uma troca de informações mais completa, por ocasião da consulta de enfermagem, fato que corrobora nossa colocação.

Nesse sentido, Fortes \& Martins (2000) ressaltam que a proposta do
${ }^{3} \mathrm{O}$ consentimento de participação do estudo foi dado por todos os sujeitos pesquisados. Foram assegurados aos mesmos, o sigilo e o anonimato. $A$ pesquisa foi aprovada pelo Comitê de Ética em Pesquisa (CEP), do Complexo Hospitalar da Universidade Federal do Ceará, na conformidade das diretrizes e normas que regulamentam a pesquisa envolvendo seres humanos, Resolução n $196 /$ 96, do Conselho Nacional de Saúde MS. Foi financiada pela Fundação Cearense de Amparo à Pesquisa (FUNCAP), sob a forma de bolsa de demand social e, para sua execução, teve apoio da Secretaria de Saúde do Estado do Ceará.

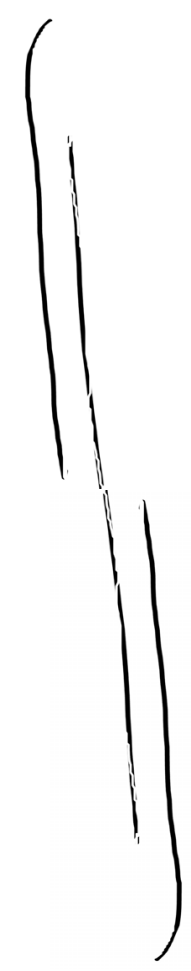


modelo assistencial atual, pautada na promoção da saúde, requer o direito do usuário à informação, de tal forma que seja este o elemento vital para a sua tomada de decisões. Assim, os enfermeiros parecem estar atuando em conformidade com o modelo de política de saúde vigente, comprometidos com a democratização do saber em saúde e com o desenvolvimento de potencialidades da clientela. Podemos enriquecer essa reflexão, subsidiadas pela idéia de King (1981), ao afirmar que, na medida em que é dever do enfermeiro informar os clientes sobre como cuidar da saúde, ajudando-os a tomar decisões conscientes, é direito dos clientes serem informados sobre os cuidados de saúde e participarem das decisões que influenciam sua vida, sua saúde e os serviços comunitários.

Analisando o contexto descrito e tomando por base dados da observação participante, pode-se afirmar que existe deficiência no planejamento das atividades de informação em saúde, no nível grupal, no sentido de que seja considerada uma atividade prioritária e realizada de forma sistemática, conforme preconizado pelo PSF, e não de forma isolada, sem um projeto de continuidade, conforme vem acontecendo. Este fato foi bastante caracterizado por ocasião do período de coleta de dados, uma vez que se teve a oportunidade de assistir, em apenas um município, a uma reunião mensal com gestantes, apesar de haver o atendimento sistemático de gestantes, nas consultas. Essa mesma constatação já havia sido feita anteriormente, por Moura Er Sousa (2002, p.1811), que identificaram déficit quanto à oferta de atividades de informação em saúde, nos mesmos municípios, atribuindo ao fato às seguintes causas:
insuficiente cobertura da população por equipes de PSF, predomínio do modelo tradicional curativo, ausência ou inadequação de local para o desenvolvimento das atividades educativas, disponibilização insuficiente de material de apoio e despreparo e desmotivação dos profissionais.

Nos depoimentos apresentados a seguir, as gestantes confirmam a escassez de atividades grupais, de informação em saúde, justo no momento em que ressaltam sua importância como parte da assistência de enfermagem no prénatal.

\footnotetext{
Que tivesse mais reunião. É pra ter toda vez que a gente tá marcada, mas às vezes elas [as enfermeiras] chegam tarde, aí as gestantes já tão tudo aqui, aí atrasa e ela já chega pra atender (G22 e G27).

Eu adoro quando a gente se reúne. Todo mundo fica se conhecendo, a gente fica sabendo quem vai ter o parto primeiro e pode até visitar e ajudar quem for tendo o filho. A gente aprende a dar de mamar, não fumar, não tomar remédio em vão (G22).
}

Da observação feita no transcorrer de uma reunião mensal, realizada durante a fase de coleta dos dados, constatou-se que esta foi conduzida de forma bastante participativa, com apoio audiovisual (álbum seriado, vídeo $e$ entrega de folder), aplicação de linguagem clara, acessível às gestantes, 
possibilitando a interação entre enfermeiras e gestantes. Sabe-se que a observação de uma só reunião grupal é insuficiente para a inferência de que os enfermeiros estão aptos a realizar adequadamente a tarefa. Comparando esses dados com o que foi observado por Moura e Sousa em 1999 (despreparo dos profissionais das equipes de PSF, médicas e enfermeiras, para promover a/ participação dos grupos; orientação pouco direcionada às necessidades de informação do grupo; uso freqüente de linguagem científica; repasse de informações desatualizadas; e utilização inadequada do material de apoio) (Moura \& Sousa, 2002), pode-se afirmar ter ocorrido um avanço. Percebe-se que as enfermeiras demonstraram compreensão aprimorada quanto ao enfoque das práticas de informação em saúde, inclusive no que se refere à adoção da metodologia participativa, valorização das necessidades de informação do grupo e utilização adequada do material de apoio.

Entretanto, Collet \& Rosso (1999) tiveram percepção diferenciada a esse respeito, ao revelarem que a prática desenvolvida por enfermeiros, em um município do interior paraense, corresponde, na maioria das vezes, a um simples repasse de informações, negando a oportunidade de se fazer uma reflexão da realidade local e das políticas oficiais de saúde. Acrescentam, ainda, que médicos, enfermeiros, auxiliares de enfermagem e agentes de saúde que atuam em PSF precisam estar cônscios da responsabilidade individual de esclarecer os usuários acerca de questões que lhes dizem respeito e criar condições para o estabelecimento de uma cultura institucional de informação $e$ de comunicação que leve em conta a condição sócio-cultural de cada comunidade atendida. Nessa perspectiva, as chances das gestantes virem a adotar medidas de autocuidado, com vistas ao alcance de metas de saúde, tornam-se mais reais.

Um estudo realizado sobre os significados e imagens do PSF, em um município do Ceará, identificou a necessidade de sistematização do componente educação em saúde, sugerindo que este seja desenvolvido por meio de uma metodologia de educação popular, formando grupos por interesses comuns, possibilitando discussões com as gestantes, simulações de práticas de atenção à criança $e$ à mulher, em uma partilha intergrupal $e$ profissional de saberes (Almeida, 2001). A mesma autora acrescenta, ainda, a importância da aproximação dos profissionais com movimentos sociais, lideranças comunitárias, grupos de interesse, conselhos, igrejas etc., no sentido de promover uma efetiva integração e participação da comunidade nas intervenções de saúde.

Considera-se oportuno discutir com mais profundidade o conceito de Educação em Saúde introduzida com o relato de Almeida (2001), como algo bem mais abrangente do que uma simples atividade de repasse de informação. Para Candeias (1997, p.210) a educação em saúde é entendida como "quaisquer combinações de experiências de aprendizagem delineadas com vistas a facilitar ações voluntárias conducentes à saúde”. A palavra delineada diferencia o processo de educação em saúde de quaisquer outras experiências acidentais de aprendizagem, ou seja, define-a como uma atividade sistematicamente planejada; a voluntariedade, por sua vez, significa sem coerção e com plena compreensão e aceitação dos objetivos educativos implícitos e explícitos nas ações desenvolvidas e recomendadas. Já Levy (2000) 
afirma que a educação em saúde deve oferecer condições para que as pessoas desenvolvam o senso de responsabilidade, tanto em relação a sua própria saúde, como em relação à saúde da comunidade, merecendo consideração como um dos mais importantes elos entre as perspectivas dos indivíduos, os projetos governamentais e as práticas de saúde. Kawamoto (1993) e Levy (2000) ressaltam, ainda, que as ações educativas em saúde são processos que objetivam capacitar indivíduos e/ou grupos, de modo que possam assumir a responsabilidade de ajudar na melhoria das condições de saúde da população. As autoras acrescentam, ainda, que os profissionais e a população devem compreender que as ações oferecidas pelos serviços de saúde, assim como o esforço da própria população, mediante conhecimentos, motivação, reflexão $e$ adoção de novas práticas de saúde, é que irão garantir a saúde da comunidade.

Diante desta reflexão vale chamar a atenção dos enfermeiros para que, ao realizarem as atividades de orientação/informação, tenham a compreensão de que estão contribuindo profundamente para a educação em saúde, valendo avançar para o alcance da educação como meta maior. A adoção de práticas benéficas à saúde, bem como a mudança para comportamentos favoráveis à saúde é o resultado esperado de um bom programa de educação em saúde.

Serão destacados, a seguir, alguns depoimentos de gestantes que fomentaram uma tentativa de avaliação do impacto das atividades de orientação/informação em saúde sobre a implementação do autocuidado por parte dessas usuárias. Os depoimentos das gestantes demonstram que a prática do autocuidado é exercida por algumas gestantes, confirmado pelas percepções das enfermeiras com relação a prática do autocuidado: 16 enfermeiros $(53,3 \%)$ consideraram que as gestantes realizam o autocuidado satisfatoriamente; 13 enfermeiros $(43,3 \%)$ consideraram que o autocuidado é exercido com limitações e um enfermeiro (3,3\%) não avaliou. Ressalta-se que esse tipo de avaliação, permitindo identificar os efeitos da orientação/informação sobre a qualidade de vida dos clientes, deve ser realizado continuamente pelos enfermeiros.

Ela [a enfermeira] orienta mais sobre a alimentação. Eu estava com muito peso. Ela [a enfermeira] mandou diminuir as massas e eu estou achando bem melhor. Pediu pra eu caminhar, estou fazendo $e$ é bom mesmo (G30).

A enfermeira orientou sobre o repouso e como evitar esforço, pois eu estava com dores, e eu cumpri o que ela mandou. Hoje eu queria que ela me orientasse sobre a inflamação [...] (G18).

Ter cuidado de repousar. Proibiu ter relação. Conversei com meu esposo e ele entendeu (G15).

Portanto, a prática de comunicação/informação em saúde pode ser desenvolvida por meio de uma proposta de sistematização da assistência de enfermagem (SAE), de maneira que sejam identificadas as principais necessidades de informação do cliente, da família e/ou da comunidade, e ao serem atendidas, possam os efeitos ser monitorados com relação ao impacto sobre o comportamento e a vida das pessoas. 


\section{Considerações finais}

O trabalho desenvolvido possibilitou constatar que a atividade de comunicação/ informação em saúde, como assistência de Enfermagem no pré-natal, começa a ser implementada de forma mais inovadora e participativa, nos municípios onde foi realizada a pesquisa, apesar da baixa freqüencia observada e da ausência de planejamento de atividades grupais, o que poderá servir para resgatar a dimensão de uma atividade sistemática e contínua. Já no nível individual, as enfermeiras vêm atuando de forma mais constante, com o reconhecimento em pesquisa de âmbito estadual.

Ademais, é necessário introduzir a prática dessas atividades (individual e grupal), de maneira a sobrepor as consultas, com a proposta de reversão do modelo tradicional biomédico (voltado à doença) para o modelo atual, que tem como foco a promoção da saúde e que, portanto, traz a democratização do saber em saúde e a avaliação de impacto sobre a qualidade de vida das pessoas como principal meta.

Pode-se considerar, ainda, o salto qualitativo que foi dado de 1999 para 2001, uma vez que se observou, no transcorrer desta pesquisa, uma experiência grupal, na qual constatou-se a introdução de técnica adequada à participação do grupo, aspecto favorável ao desenvolvimento de potencialidades para o exercício do auto-cuidado, da promoção da saúde e do estímulo à cidadania.

Conclui-se que o desenvolvimento de atividades de comunicação/informação em saúde na assistência ao pré-natal merecem ser priorizadas, planejadas e desenvolvidas com vistas a gerar mudanças de comportamentos, pela adoção de práticas sistemáticas e participativas por todos os profissionais que integram as equipes de PSF.

\section{Referências}

ALMEIDA, M. Programa Saúde da Família: significados e imagens. 2001. Dissertação (Mestrado). Faculdade de Farmácia, Odontologia e Enfermagem, Universidade Federal de Fortaleza. Ceará.

BRASIL. MINISTÉRIO DA SAÚDE. Assistência pré-natal: manual técnico. Brasília: Ministério da Saúde, 2000.

CANDEIAS, N. M. F. Conceitos de educação e de promoção em saúde: mudanças individuais e mudanças organizacionais. Rev. Saúde Pública, v.3, n.1, p.209-12, 1997.

CEARÁ. SECRETARIA DE SAÚDE DO ESTADO. II Análise situacional da saúde reprodutiva no Ceará: avaliação de impacto 1993-1997. Fortaleza: Secretaria da Saúde, 1998.

COLLET, N.; ROSSO, C. F. W. Os enfermeiros e a prática de educação em saúde em municípios do interior paranaense. Disponível em: <http://www.fen.ufg.br/revista>. Acesso em: 17 out. 1999.

CRUZ NETO, O. O trabalho de campo como descoberta e criação. In: MINAYO, M. C. S. (Org.). Pesquisa social: teoria, método e criatividade. 4.ed. Petrópolis: Vozes, 1995, p.51-66.

FORTES, P. A. C.; MARTINS, C. L. A ética, a humanização e a saúde da família. Rev. Bras. Enferm., v.8, n.53, p.31-3, 2000.

KAWAMOTO, E. E. Educação em saúde. Enfermagem comunitária. EPU, v.3, p.29-33, 1993.

KING, I. M. A theory for nursing: systems, concepts, process. New York: Wiley medical publications, 1981. 
LEVY, S. Programa educação em saúde. Disponível em: <http://www.saúde.gov.br/programas/pes/ pes/index.htm>. Acesso em: 05 out. 2000.

MOURA, E. R. F. Assistência de Enfermagem no pré-natal no contexto do Programa Saúde da Família. 2001. Dissertação (Mestrado), Faculdade de Farmácia, Odontologia e Enfermagem, Universidade Federal de Fortaleza. Ceará.

MOURA, E. R. F.; SOUSA, R. A. Educação em saúde reprodutiva: proposta ou realidade do programa saúde da família? Cad. Saúde Pública, v.18, n.6, p.1809-11, 2002.

NAVAJAS FILHO, E. Os processos de identificação e introjeção na gravidez. In: ZUGAIB, M.; TEDESCO, J. J. A.; QUAYLE, J. (Org.). Obstetrícia psicossomática. São Paulo: Atheneu, 1997. p.10-4.

TRIVIÑOS, A.N.S. Introdução à pesquisa em ciências sociais. São Paulo: Atlas, 1993.

TYRREL, M. A.; CARVALHO, V. Programas nacionais de saúde materno-infantil: impacto político social e inserção de enfermagem. Rio de Janeiro: UFRJ, 1995.

VARELA, Z. M. V. Habilidades femininas no agir didático. In: VARELA, Z. M. V.; SILVA, R. M.; BARROSO, M. G.T (Org.). Ensino na universidade: integrando graduação e pós-graduação. Fortaleza: FCPC, 2000. p.17-25.

MOURA, E. R. F.; RODRIGUES, M. S. P. Comunicación e información en Salud en el examen prenatal, Interface - Comunic, Saúde, Educ, v.7, n.13, p.109-18, 2003.

Se trata de una investigación que tuvo por objetivo analizar las actividades de comunicación / información en salud, implementadas en el transcurso de la atención prenatal, por enfermeros que actúan en el Programa Salud de la Familia, Brasil (PSF). Los datos fueron recolectados en ocho municipios del Estado de Ceará, de mayo a junio de 2001, utilizando las técnicas de observación participante, entrevista y cuestionario. La muestra fue constituida por trinta enfermeros y trinta gestantes. Se constató que las actividades de comunicación / información en salud comienzan a ser implementadas de forma más innovadora y participativa, en el universo investigado, no obstante con baja frecuencia. Se identificó ausencia de planificación de las actividades grupales, perjudicando su ejecución de manera sistemática y continua. En el ámbito individual, los enfermeros están desarrollando acciones de comunicación / información como parte de la rutina de la atención de la enfermería a las gestantes, incluso con sus desempeños reconocidos en el ámbito provincial. Se recomienda que esas actividades sean implementadas de manera que sobrepongan las consultas, con la propuesta de contribuir a la implantación de un modelo de promoción de la salud (PSF).

PALABRAS CLAVE: Atención prenatal; atención primaria de la salud; promoción de la salud; Educación en Salud; communicación. 
MOURA, E. R. F.; RODRIGUES, M. S. P.
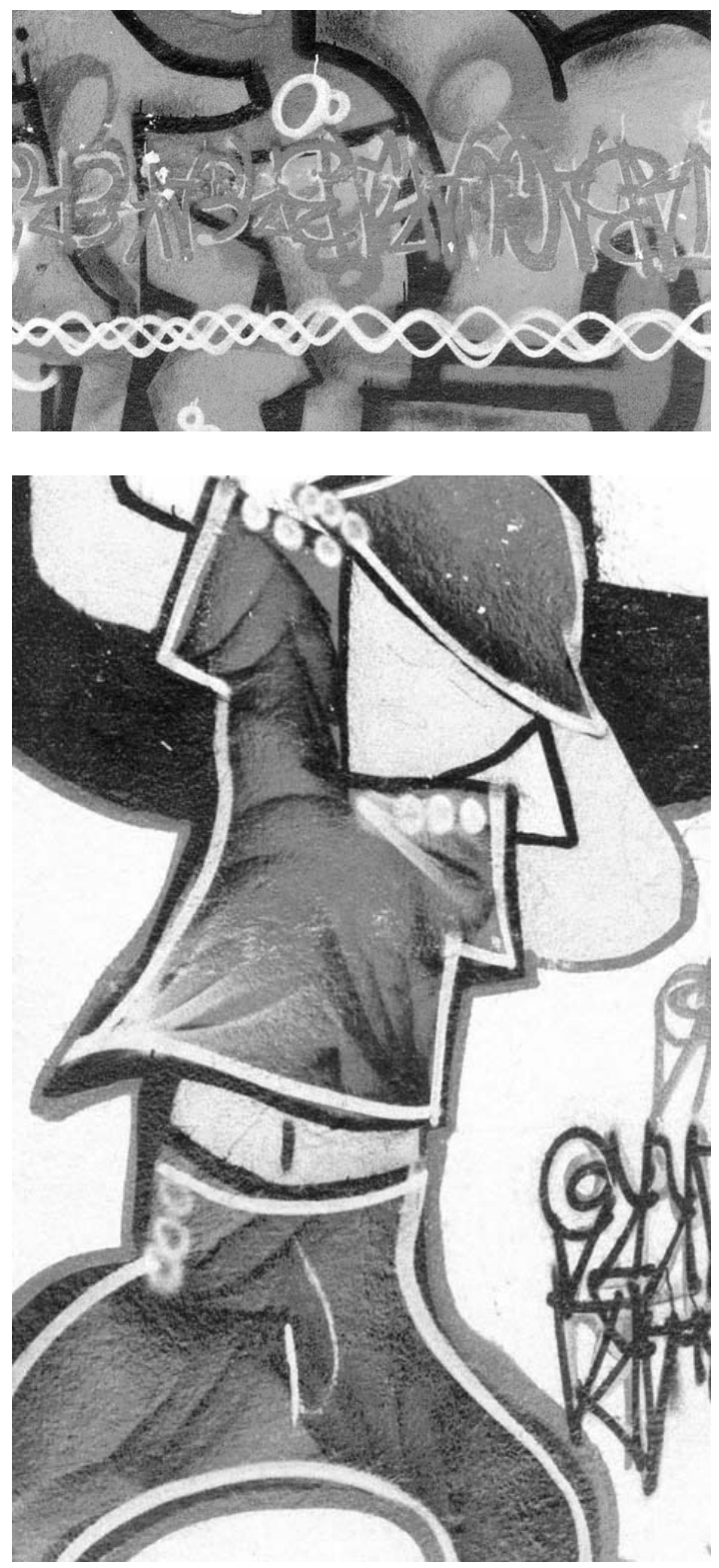

MARIA DO SOCORRO FIGUEIREDO, 2003 